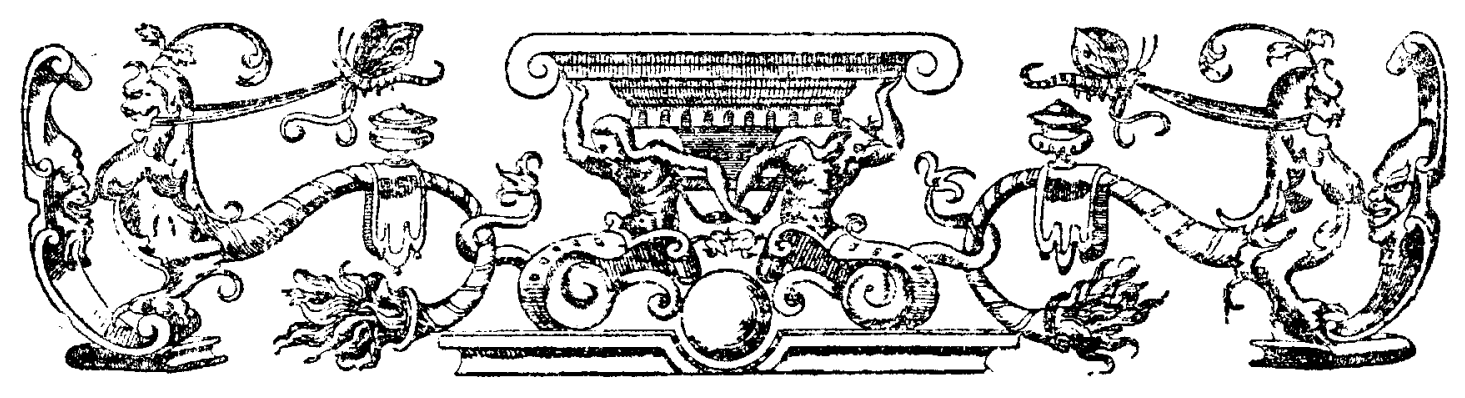

\title{
Ein gleichzeitiges Reiterstandbild des deutschen Königs Wilhelm (von Holland).?
}

vox Prof. Dr. F. PHILIPPI (Münster).

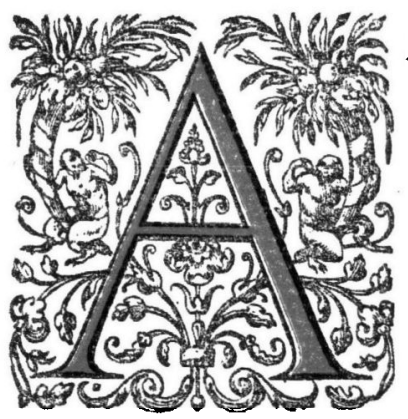

II (vom Beschauer aus gesehen) linken Vierungspfeiler des St. Georgen-Chors im Bamberger Dome steht das bekannte Reiterstandbild, dessen Deutung schon manche Feder beschäftigt hat. GEORG DEHIo hat sich in seiner ausgezeichneten Geschichte der deutschen Kunst und wohl zuletzt, mit dem Kunstwerke befasst und will in ihm den heiligen Georg, den Mitpatron der Bamberger Kirche erblicken. 1)

Ich möchte im Folgenden eine andere Deutung versuchen, weil ich in dem Bild uberha:ıt keinen Heiligen zu sehen vermag, wenngleich zuzugeben ist, dasz der Platz, an welchem es aufgestellt ist, nach mittelalterlicher Auffassung und Sitte am ehesten einem Heiligen gebührte. Mich bewegt allerdings zu dieser Stellungnahme weniger der Mangel des Heiligenscheins, des Nimbus', dessen sich ja überhaupt die Ramberger Künstler jener Zeit bei freistehenden Figuren nie, aber auch bei Relieffiguren nicht immer zur genaueren Kenizeichnung ihrer

1) Abbikingen häufig; zuletzt bei Defro a.a. O. I Abbildungsband No. 454-455; Max SAUkri.AnDr, „Deutsche Plastik des Mittelalters" 34, 35; sowie aut der letzten Ausgabe der Reichsbanknoten zu 100 M. 
Figuren bedienen, als vielmehr die durchaus weltliche Haltung der Figur und ihr nicht, wie es einem Heiligen ziemen würde, frommer und sinnender, sondern durchaus weltlicher, trotziger und überhebender Gesichtsausdruck. Auf keinen Fall aber möchte ich der Deutung aut den hl. Georg, den Ritterheiligen, das Wort reden, wenn sie auch sachlich, wie oben angedeutet, sehr nahe liegt, weil dieser Heilige durchaus gerüstet und meist den Drachen besiegend dargestellt zu werden pflegt, während die in Frage stehende Figur keinen Panzer trägt, ja sogar, wie es wenigstens scheint, nicht einmal ein Schwert angelegt hat.

Dagegen trägt der Bamberger Reiter einen Kopfschmuck, welchen man bei Bildern des Drachentodters wohl kaum jemals antreffen wird: eine Krone. Diese allerdings einfache Zackenkrone aber kennzeichnet in der Kunst der I3. Jahrhunderts, aus welchem nach ubereinstimmender Schätzung das Bild stammt, unbedingt einen $\mathrm{König.}$

Wir haben also in der Figur ohne Zweifel einen König zu sehen. Es handelt sich nun um Entscheidung der Frage, ob der Künstler einen König aus der heiligen Geschichte oder einen weltlichen Herrscher seiner eigenen Zeit hat darstellen wollen oder sollen.

Die Zahl der Könige, welche in der heiligen Geschichte vorkommen, ist nicht grosz. Am häufigsten werden als Könige genannt und dargestellt die drei Magi aus dem Morgenlande; wirklich ist, soviel ich sehe, jemand darauf verfallen, in dem berittenen Könige an der Schwelle des Bamberger Domchors einen dieser frühen Anbeter des Jesus-Kindes zu erblicken. Da aber diese heiligen Reisenden sehr selten auf ihren Pferden im Bilde erscheinen, l) sondern entweder auf die Gottesmutter zueilend oder vor ihr knieend, während die Diener in ehrfurchtsvoller Entfernung die Reittiere halten, kann ich mich mit einer solchen Ausdeutung um so weniger befreunden, als auch sie mit dem Ausdrucke im Gesichte des Reiters schier unvereinbar erscheint. Aber ich glaube auch nicht, dasz die Figur einen anderen König aus der heiligen Geschichte darstellt, wenigstens finde ich weder in der Kleidung und Haltung des Körpers noch im Ausdrucke des Kopfes eine Hindeutung auf den König DAvid, den Vierfürsten HERodes noch einen anderen alten Herrscher, zumal jede Beigabe, wie z.B. bei David die Harfe, fehlt.

Ich sehe mich daher zu der Annahme gezwungen, dass wir es mit einem weltlichen Herrscher aus dem Mittelalter zu tun haben; dabei wäre am ersten gerade in Bamberg an Kaiser HeINRICH II, den Stifter des Bistums zu denken, zumal er ja in die Schaar der Heiligen aufgenommen worden ist. Aber auch

1) Sie sind vereinzelt auf der Reise dargestellt in dem Augenblicke, in welchem ihnen ier Stern von Bethlehem erscheint. Der Ausdruck auf diesen Bildern ist aber ein dem Ausdrucke des Reiters vollkommen entgegengesetzter. 
segen diese Deutung sprechen eine Reihe von Beobachtungen, welche das Bild selbst uns aufdrängt. Weder die ausgesprochene Jugendlichkeit des Kopfes, noch sein Ausdruck, noch die ganze Haltung der Figur lassen sich als bezeichnend für den frommen Gründer des Heiligtums erkennen. Wie man ihn sich in der Mitte des I 3 Jahrhunderts vorstellte, beweist sein Bildnis an der Wandung des Ostportals wo er mit seiner Gemahlin KUNEGUNDE dargestellt ist: älter, würdiger, bärtig, in frommem Sinnen, in den Händen Scepter und Reichsapfel, und auf dem Haupte die gleiche Krone, wie sie der Reiter trägt. 1)

Da nun die ganz individuellen Zuge in dem Kopfe des Reiters dazu drängen in ihm das Bildnis einer bestimmten Persönlichkeit, eines bestimmten Königs zu erblicken, bleibt kaum etwas anderes übrig, als in ihm einen Herrscher aus der unmittelbaren Umwelt des Künstlers zu vermuten d.h. also einen letzten Stauten oder einen ihrer Gegenkönige. ${ }^{2}$ )

Von den letzten Staufen musz wohl schon aus rein äusserlichen Gründen FRIEDRICH II ohne weiteres ausscheiden, da für ihn die Züge des Bildes zu jugendlich erscheinen, und dasselbe ist wohl von seinem Gegenkönige HEJNRICH RASPE zu sagen, der um 1202 geboren ist, also zur Zeit der Herstellung des Bildes um 1250 schon ein gereifter Mann war $(t$ 1248). Dagegen könnte man unter Berüclisichtigung dieses Hauptkennzeichens des Kopfes ebenso wohl an König KonRAd IV wie an Wilhelm voN Holland denken, weiche beide r 228 geboren waren und beide vor Vollendung ihres dreiszigsten Lebensiahres den Tod fanden.

Was sagt nun diesen Erwägungen gegenüber, wic sic die oberflächliche Betrachtung an die Hand gibt, die schriftliche Ueberlieferung? Sie ist dürftig genug, nicht nur für die ganze Zeit, sondern auch vor Allem für die Beantworiung der hier gestellten Frage. Weder die Todrenbücher ${ }^{3}$ ) der Kirche noch die erzählenden Quellen geben irgend eine Nachricht über nähere Beziehungen einer dieser Herrscher oder ins besondere des jungen Grafen von Holland zum Bamberger Domkirche oder gar zu ihrem Bau.

1) Vergl. Max Sauerfanin, "Deutsche Plastik des Mittelalters" 22 und 252 sowic bei G. Dehro a. a. $O$. Abbildungen No. 456 neben dem Kopfe des Reiters.

2) Hierbei ist allerdings noch die Frage aufzuwerfen, ob es dem Bildhaner möglich gewesen sein wird, dieses erstrebte Ziel auci tatsächlich zu erreichen, indem er wirklich den Künig, den er darstellt, selbst als Vorbild benutzte. $E_{s}$ ist $i_{a}$ auch der Fall dentbar, dass er ein ihm geeignet erscheinendes Modell benutzt hätle, welches auf dem Leibe eines Niedriggeborenen gesessen hätte, aber die gewünschten Züge und den gewiinschten Ausdruck viedergab. Diese Möglichkeit muss gewiss bei der Abschätzung der Sicherheit der hier ge:̈usserten Vermutungen mit berücksichtigt werden. Weiter scheint mir jedoch ihre Bedeutung nicht zu reichen.

3) Abredruckt in "Siebenter Bericht iber das Bestehen und Wirken des hist. Vereins zu Bambe:"g" Bamberg 1844 , S. $67-319$. Der letzte darin genannte Iönig ist Philipp von Schwaben. 
Nur die Geschichte des um die Mitte des I 3 Jahrhunderts (1242 - I 257) das Stift verwaltenden Bischofs HEINRICH I, l) gewährt einigen Aufschluss über seine persönlichen Beziehungen zu den gleichzeitig regierenden Königen, aber dadurch erscheint die Entscheidung für den einen oder den andern dieser Herrscher eher erschwert als erleichtert zu werden, denn dieser Kirchenfürst war zwar eine Kreatur FriedrichS II, der ihn schon frühe (I232) in Catania auf den Bischofsstuhl zu bringen versuchte, ihm später die Propsttei am Krönungsstifte in Aachen verschaffe und 1242 behiilfich war an Stelle des wegen schlechter Wirtschaft abgesetzten Bischofs POPPO voN MERAN auf den Bamberger Bischofsstuhl zu gelangen. Er war gelehrter Jurist (Magister) und in des Kaisers Kanzlei erst als Notar, schliesslich als Protonotar tätig. Mehrfach vertrat er seinen Herrn in wichtigen diplomatischen Geschäften: so beschwor er den Vertrag mit König Ludwig IX von Frankreicir 1232 in die Seele des Kaisers.

Aber er wusste seinem Gönner schlechten Dank. Als der Pabst den Kaiser bannte und absetzte, verliess ihn Bischof HeINRICH im Herbste I 245 und war, wenn auch nicht in vorderster Reihe, so doch als Vermittler sowohl bei der Erhebung Heinricis RASpe wie Wilhelas voN HOLLAND zu Gegenkönigen beteiligt. In wie weit er in die Verschwörung gegen diesen letzten Herrscher im Jahre 1255 , verwickelt war, und ob diese Bewegung uberhaupt in das Reich der Tatsachen gehört, ist hier nicht weiter zu untersuchen. Aber das musz noch betont werden, dass er zeitweise zu dem Sohne FrIedrichs II, dem jungen Könige KONRAD IV in nahen Beziehungen gestanden hat, denn dieser Herrscher hat ihm - im Auftrage seines Vaters - im Jahre 1243 zahlreiche Gunstbezeugungen zukommen lassen und ihn im Dezember desselben Jahres iange an seinem Hofe gesehen, wie sein Vater früher so manches Mal.

Dagegen lassen sich vON HEINRICH RASPE nur eine, von WILHELM von IJOLLAND aber keine einzige zu Gunsten des Stiftes BAMBERG und seines Bischofs erlassene Urkunde nachweisen.

Dieser Befund könnte nun zu der Vernutung veranlassen, dennoch einen der letzten Staufen in dem königlichen Reiter zu erblicken. Aber bei näherer Ueberlegung wird man davon zurückkommen müssen. Dass FRIEDKICH II wohi nicht in Frage kommt, wurde schon oben mit Rücksicht auf die Jugend des Darge-

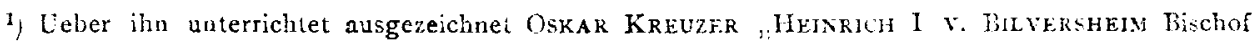
von Bamberg (1242 - 1257) I - III Hamberger Gymnasialprogramme 1907 - I909 nach desselben Verfassers" Regesten des Bamberger Bischofs H. I. v. B. Schweinfurter Gymnasialprogramm igor. Für Ucbernittlung dieser Schriften und weitere freundliche Fürderung sage ich den Bayerischen Staatsarchive in Bamberg verbindlichsten Dank. 


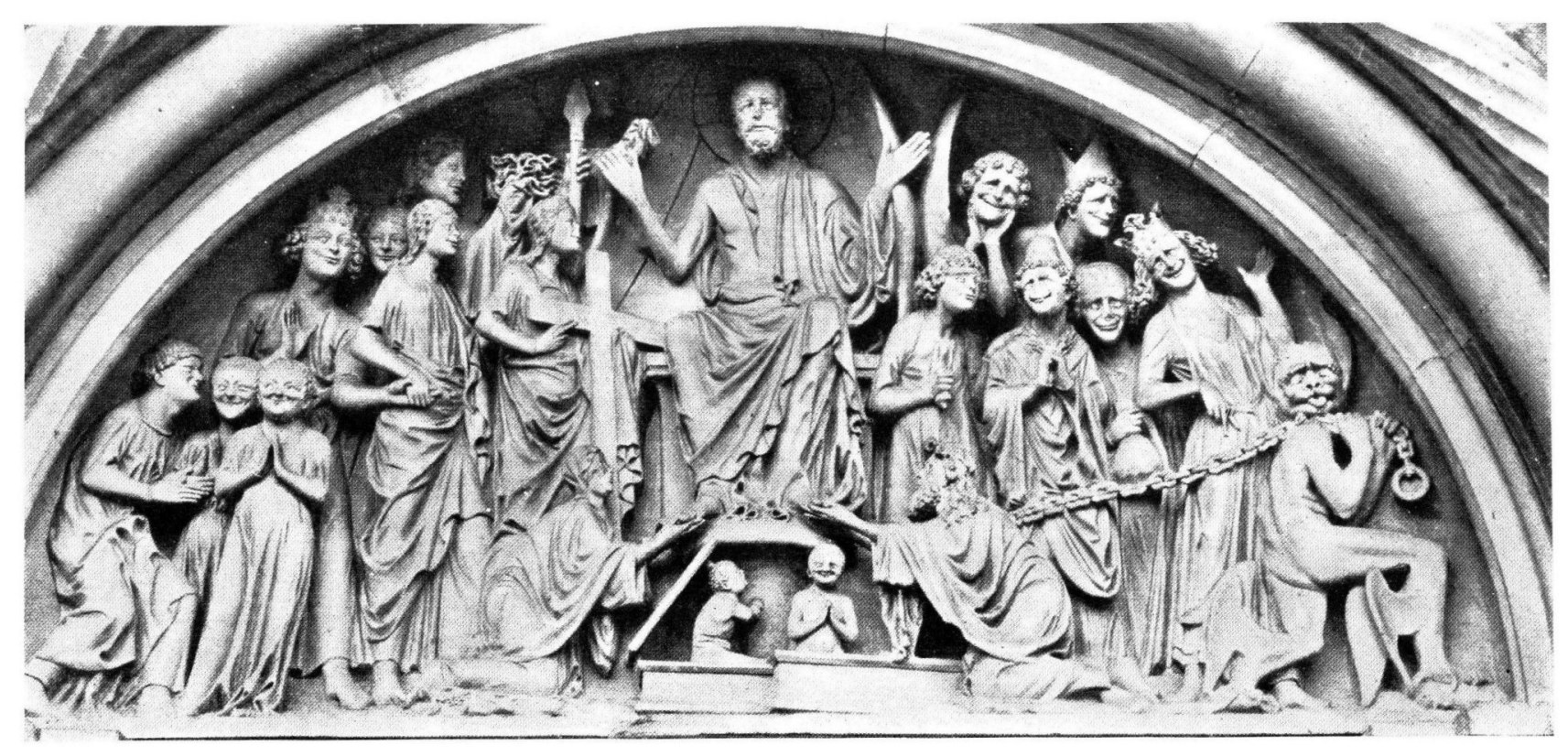

füngste Gericht, Himmel u. Höllefahrt (Dom zu Bamberg).

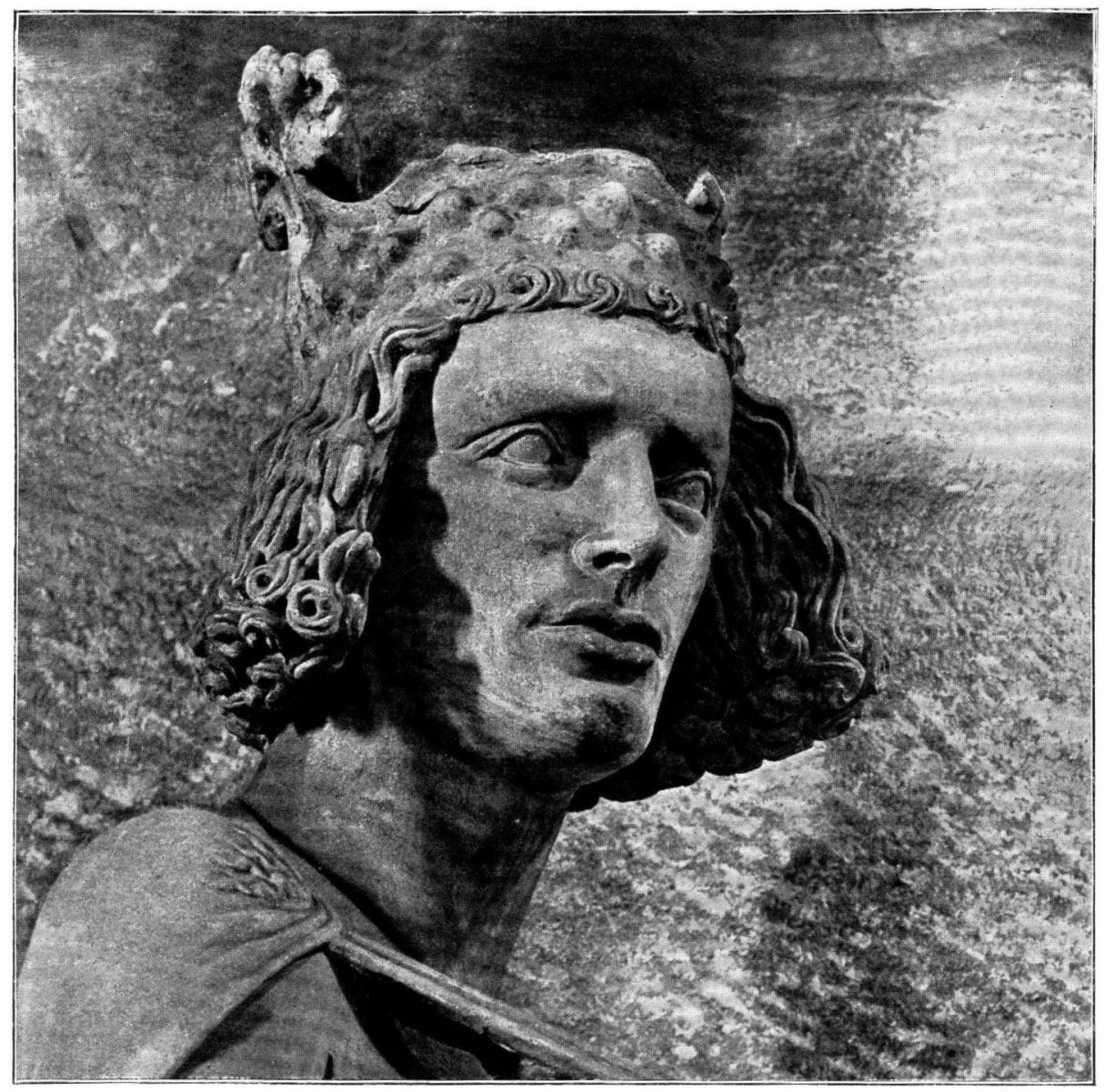

Kopf des Bamberger Reiterstandbildes. 
stellten gesagt. Zudem passen die Züge und der Ausdruck in keiner Weise zu dem Bilde, welches man sich nach seinen Siegeln machen muss. 1)

Ein viel gewichtigerer aber und die Annahme, dass einer der letzten Staufen in dem Bamberger Reiter dargestellt sei, geradezu verbietender Umstand ist der, dass sie gebannt waren, und dass ihre Bilder also, wenn sie vielleicht aus Dankbarkeit im Dome zu Bamberg aufgestellt gewesen sein könnten, ohne Zweifel nach ihrem Abfalle von der Kirche und ihrer Verurteilung durch die Kirche von einem Bischofe, der nach seiner Umkehr bis au sein Lebensende unentwegt auf der Seite des Papstes stand, wieder entfernt worden wären. Dasz dieses für Bamberg unbedingt angenommen werden muss, geht auch noch aus der Beobachtung hervor, dass FRIEDRICH II an einer anderen Stelle des Domes, wie unten nachzuweisen ist, als vom Teufel mit Judas zusammen zur Hölle geschleppt dargestellt sich findet.

So darf man also wohl nur die beiden Gegenkönige HeINRICH RASPE und WilHelm voN HollaNd in Betracht ziehen. Dass bei der Wahl zwischen diesen Beiden das Äuszere des Bildes in sciner unbedingten Jugendlichkeit tür WILHELM und gegen HEINRICH spricht ist schon oben angedeutet. Ausserdem aber scheint das Verhältnis des Bisschofs Heinrich zum Könige HFinrich trotz der einen oben erwähnten Urkunde, welche ubrigens auch keiren unzweifelhaften Gnadenbeweis darstellt, kein ungetrübtes gewesen zu sein. Der Kirchenfürst hatte sich Hoffnung gemacht, vom Könige ais Kanzler angenomen zu werden; statt dessen fiuden wir den erwählten Bischof HEINRICH vON SPEYER in dieser Stellung.

So verengt sich also der Kreis der für die Deutung des Bamberger Reiters in Frage kommenden Personen immer mehr, und es bleibt unter den Königen kaum ein andrer als WILHELM VON HOLLAND äbrig. Dass zu ihm die Jugendlichkeit des Kopfes vorzüglich geht ist schon oben hervorgehoben. Es ist ein in den 20 Jahren stehender Jüngling, der uns in dem Bildnisse gegenübertritt. Aber auch der Ausdruck des Gesichts im Einzelnen stimmt vortrefflich zu den Charakterschilderungen, die wir von dem jungen Holländer Grafen überkommen haben.

Denn wir sind bei diesem Fürsten in der ausnahmsweise glücklichen Lage, zwei Charakteristiken von ihm zu besitzen, die uns der Reimchronist MELIS STOKE hinterlassen hat. Er war zwar nicht ein Zeitgenosse WILHELMS, sondern ein bis zwei Menschenalter jünger. Seine Nachrichten über WILHELM geiten aber für unbedingt zuverlïssig, weil man wohl mit Recht annimmt, dass er Mitteilungen

r) Verol. meire Darlegungen in ,Zeitschrift f. bilkende Kunct" N.F. XV S. 857 . Out-IIlland $192 \mathrm{r}$. 
von Zeitgenossen des Königs unmittelbar erhalten und verwertet hat. Er sagt an der einen stelle:

"Hochtrabend war er und sehr stolz,

So dasz er Herr der Herren hiesz"

und an der anderen:

"Der Graf war jung, stolz und rauh

Und alle Falschheit war ihm leid"

Mir scheint man kann den Gesichtsausdruck des Bambergers Reiters kaum bezeichnender als durch diese Verse wiedergeben, und umgekehrt können diese Reimzeilen kaum packender als durch das Standbild erläutert werden. 1)

Eine weitere Bestätigung für die Annahme, dass das Bild König WilHelM von Holland darstellt, bietet des Königs Siegel. s) Es ist freilich sehr schematisch in Tracht und Haltung, aber es zeigt doch ein glattrasirtes Gesicht und eine auffallend mächtige Lockenperücke, die zwar nicht so steif abstehend frisirt erscheint, wie an dem Bilde, dennoch aber im Vergleiche zu den Siegeln seines Vorgängers HEINRICH RASPE, und seines Nachfolgers RICHARD VON KORNwALLIS ${ }^{3}$ ) als besonders üppig Erwähnung verdient.

Nun ist aber dieser Königskopf am Bamberger Dome nicht nur auf den Schultern des Reiters am Chore zu sehen, sondern er kehrt auch in einer höchsteigenartigen Darstellung des jüngsten Gerichtes in der Bogenfülling der Ostpforte wieder, wenn auch mit ganz andrem Ausdruck. 4)

Wir sehen auf diesem Bilde GotTVATER mit erhobenen Händen auf einem Throne sitzen, zu seinen Füssen öfnen sich zwei Gräber, rechts und links knieen vor ihm Maria und Christus durch die Dornenkrore gekennzeichnet; sie umfassen, Erbarmen flehend, seine Füsse. Den Grund füllen rechts und links die Gruppen der Seeligen und der Verdammten. In beiden bildet die Hauptfigur ein gekrönter König. In der Gruppe der Seeligen ein junger, freudig erstaunt blickender mit den Zugen des Reiters am Chor; in der Schaar der Verdammten ein alter, jammernd und wehklagend. An ihn drängt sich JudAs mit dem Beutel der 30 Silberlingen, dem Geistliche folgen. Alle zusammen schleppt ein grinsender, an den Beinen geflugelter Teufel fort aus dem Angesichte GotTVaters, sie mit einer Kette umfassend, während ein Engel mit dem Schwerte sie vom Herm des Himmels

1) W. G. Brill, Rijmkroniek van MELIs Sroke in .Werken van het Historisch Gencotschap, gevestigd te Utrecht", Nieuwe Serie No. 40 und 42 . Die Stellen Bd. 40 S. I64 Z. 723, 724: Iloochdraghende was hie ende stout sere, sodat hie hiet der heren here, und ebenda S. I68 Z. S03, 804: Die grave was stout, jonc ende wreet ende alle valscheit had hie leet.

2) O. Posse, die Siegel der deutschen Kaiser $3 \hat{5}, 4$.

3) O. Possi: a.2. O. 34, I und 37, 3.

4) M. Sauerlandt a. O, 21. 
scheidet. Die Seeligen aber zur Rechten bringen mit glucklicher Gebärde dem Weltenrichter, die Marterwerkzeuge, Kreuz, Dornenkrone, Lanze und Schweisstuch seines Sohnes entgegen, um auf Grund dieser Fürsprache Erlösung und Begnadigung zu erlangen. Unter ihnen, dem Träger des Kreuzes folgend, führt eine jugendliche, glaubensstolz einherschreitende Frauengestalt den jungen König heran.

Es bedarf wohl keiner weiteren längeren Darlegung, dasz hier die Höllenfahrt des gebannten Kaisers FRIEURICH und die Aufnahme seines jugendlichen Gegenkönigs, also WILHELMs voN HoLlaNd in den Himmel dargestellt ist. Und dieser junge König hat, wie schon oben angedeutet, die Züge des Reiters am Chor.

$\mathrm{Da}$ die beiden Könige dieses Flachbildes als verstorben gedacht sind, wird man es wohl nach 1256 dem Todesjahre WiLHELms setzen müssen. Ob aber das Reiterbild nicht doch einige Jahre fruher entstanden ist, mag dahingestellt bleiben.

Ist es nun eine Ironie des Schicksals oder ein Zufall, dasz das Bild des "Pfaffenkönings" zum Sclimuck der Banknoten des deutschen Reiches in den Tagen seiner tiefsten Erniedrigung gebraucht worden ist. Ich wage hier diesen Gedanken nicht weiter nachzugehen.

Die Abbildungen sind nach Aufnamen der Fima $G$. HaAF in Bamberg (jüngstes Gericht) und Dr. F. StödTNer in Berlin N.W. gefertigt. Beiden Firmen sei fïr die freunolichste erteilte Erlaubuiss zur Benntzungr ihrer Verlagsgegenstände Dank gesagt. 